\title{
No-wall holographic model for QCD
}

\author{
S. S. Afonin \\ V. A. Fock Department of Theoretical Physics, Saint-Petersburg State \\ University, 1 ul. Ulyanovskaya, 198504, Russia
}

\begin{abstract}
We propose a new type of bottom-up holographic model describing the Regge like spectrum of mesons. This type of spectrum emerges due to condensation of a scalar field in the bulk anti-de Sitter space. The gauge invariance of the action describing the vector mesons requires that the bulk scalar field must correspond to an operator of canonical dimension two. This is not necessary for the description of the scalar sector where the generation of the mass spectrum can be directly related to the formation of gluon condensate in QCD. It is also demonstrated that such an explicit introduction of local dim2 operator into the model leads to disappearance of the corresponding contribution from the dim2 condensate in the power expansion of the gauge-invariant vector correlator as required by QCD.
\end{abstract}

\section{Introduction}

The bottom-up holographic models inspired by the ideas of AdS/CFT correspondence [1,2] turned out to be an interesting phenomenological approach to the physics of strong interactions encompassing in a uniform framework the low-energy effective field theories and the QCD sum rules in the large- $N_{c}$ limit. The simplicity of theoretical setup represents a nice feature of holographic approach to QCD. At the same time, the setup of many AdS/QCD models looks ad hoc and motivated by the phenomenology rather than by some consistent logic. In spite of extensive literature on this subject appeared in the last few years (an incomplete list is given by Refs. [3 23]) some important questions about the foundations of holographic models remain unanswered. One of these questions is the origin and physical meaning of the dilaton background in the action of the so-called soft-wall models which were introduced in Ref. [5, 6] to describe holographically the Regge-like spectrum. The corresponding background $e^{ \pm \Lambda^{2} z^{2}}$ (we will refer to such soft-wall models as $\mathrm{SW}^{ \pm}$) violates explicitly the Lorentz invariance along the fifth coordinate $z$ and it looks therefore puzzling how it could arise from a consistent solution of five-dimensional Einstein equations (see, however, the toy model of Ref. [12]). On the other hand, this background provides the Regge-like 
spectrum for the radially excited states, $m_{n}^{2} \sim \Lambda^{2} n$, introducing simultaneously the mass scale $\Lambda$. In the present work, we will try to shed light on the problem by transforming the SW model into a holographic model with a dynamical mechanism for mass generation based on condensation of a scalar field. Such a "no-wall model" turns out to differ from the original SW model representing rather a mean of the $\mathrm{SW}^{+}$and $\mathrm{SW}^{-}$models. This property leads to interesting consequences, for instance, the dimension-two condensate in the power expansion of vector correlator is absent automatically in the no-wall model.

The paper is organized as follows. The no-wall holographic model is introduced in Section 2. In Section 3, we calculate the two-point vector correlator and clarify the emerging difference between the SW and no-wall models. Our conclusions are summarized in Section 4.

\section{The no-wall model from the soft-wall model for vector mesons}

Consider the simplest action of the $\mathrm{SW}^{ \pm}$model for vector mesons without the chiral symmetry breaking term,

$$
S=\int d^{4} x d z \sqrt{g} e^{ \pm \Lambda^{2} z^{2}}\left\{-\frac{1}{4 g_{5}^{2}} F_{M N} F^{M N}\right\},
$$

where $F_{M N}=\partial_{M} V_{N}-\partial_{N} V_{M}, M=0,1,2,3,4$. One writes usually the action (1) in terms of the left and right fields, $F_{L}^{2}+F_{R}^{2}$, this will be not essential for us, so the parity of $V_{M}$ is not fixed at this stage. For simplicity, we do not introduce also the isospin index, i.e. the action (11) is supposed to describe the isosinglet vector states, say, the $\omega$-mesons. Our analysis, however, will be valid for all light vector mesons ( $\rho, a_{1}$ etc.) since we are interested in the mass spectrum, hence, only the quadratic terms in (1) are relevant. The coupling $g_{5}$ plays the role of normalization constant for the $5 \mathrm{D}$ vector field $V_{M}$ and can be fixed after calculation of the two-point vector correlator (see relation (26) below). The UV boundary value $V_{M}(x, 0)$ is interpreted as the source for a QCD operator interpolating the vector mesons, e.g., for $\bar{q} \gamma_{\mu} q$ if we consider the $\omega$-mesons. The metric is the usual AdS one,

$$
d s^{2}=\frac{R^{2}}{z^{2}}\left(d x_{\mu} d x^{\mu}-d z^{2}\right), \quad z>0,
$$

here $R$ is the radius of the AdS space and $z$ is the holographic coordinate. According to the prescriptions of the AdS/CFT correspondence [1,2], the 
masses of fields in the dual theory defined on the $\mathrm{AdS}_{5}$ space are

$$
m_{5}^{2} R^{2}=(\Delta-J)(\Delta+J-4)
$$

where $\Delta$ is the canonical dimension of the corresponding $4 \mathrm{D}$ field theory operator and $J$ is the spin. In our case, the field $V_{M}$ is massless. This field is considered as the gauge one, so we may use the gauge freedom to fix the axial gauge, $V_{z}=0$, which is implied in what follows.

The Lorentz invariance along the $z$-direction is explicitly violated in the action (11) due to the dilaton background. Let us remove this background with the help of the transformation

$$
V_{M}=e^{\mp \Lambda^{2} z^{2} / 2} \tilde{V}_{M} .
$$

The action becomes equivalent to

$$
S=\int d^{4} x d z \sqrt{g}\left\{-\frac{1}{4 g_{5}^{2}} \tilde{F}_{M N} \tilde{F}^{M N}+\frac{\Lambda^{4} z^{4}}{2 R^{2} g_{5}^{2}} \tilde{V}_{M} \tilde{V}^{M}\right\}
$$

where instead of the $z$-dependent background we have now a $z$-dependent mass term. The action (5) can be regarded as the definition of new holographic model formulated in terms of the field $\tilde{V}_{M}$ (we will omit tildes in what follows). The $z$-dependent mass contribution breaks the invariance with respect to the local gauge transformations of the field $\tilde{V}_{M}$ that corresponds to violation of a global flavor symmetry in the equivalent $4 \mathrm{D}$ field theory together with the breaking of the scale invariance ( $z$ is the inverse energy scale). There is, however, a way to formulate the model in a gauge-invariant manner: The appearance of the $z$-dependent term may be interpreted as an effect of condensation of some bulk scalar field which is coupled to the vector field via the covariant derivative,

$$
S=\int d^{4} x d z \sqrt{g}\left\{\left|D_{M} \varphi\right|^{2}-m_{\varphi}^{2} \varphi^{2}-\frac{1}{4 g_{5}^{2}} F_{M N} F^{M N}\right\}
$$

where $D_{M}=\partial_{M}-i V_{M}$. Actually, the action (6) is in one-to-one correspondence with the simplest holographic setup describing the chiral symmetry breaking [3,4]. The difference consists in the interpretation of the bulk scalar field $\varphi$. The equation of motion for $\varphi$ in the absence of the vector field $V_{M}$ is

$$
-\partial_{z}\left(\frac{\partial_{z} \varphi}{z^{3}}\right)+\frac{m_{\varphi}^{2} R^{2} \varphi}{z^{5}}=0 .
$$

In order to have the same equation of motion for $V_{M}$ that would follow from the action (5) the Eq. (7) must lead to the solution $\varphi_{0} \sim z^{2}$. This dictates 
$m_{\varphi}^{2} R^{2}=-4$. The relation (3) tells us that the field $\varphi$ corresponds then to the operator of canonical dimension $\Delta=2$. Such a gauge-invariant local operator does not exist in QCD. Nevertheless, there are many suggestions in the literature [24] that the corresponding gluon operator is required in the phenomenology for an effective parametrization of various non-perturbative effects.

It should be mentioned that if we describe the non-gauge fields the introduction of $\operatorname{dim} 2$ operator is not necessary. For instance, one may write the following "no-wall model" for the scalar mesons

$$
S_{\text {scal }}=\int d^{4} x d z \sqrt{g}\left\{\left(\partial_{M} S\right)^{2}-m_{s}^{2} S^{2}-\lambda \tilde{\varphi} S^{2}+\left(\partial_{M} \tilde{\varphi}\right)^{2}-m_{\tilde{\varphi}}^{2} \tilde{\varphi}^{2}\right\} .
$$

To have the Regge-like spectrum from the equation of motion for $S$ the field $\tilde{\varphi}$ must have the bulk expectation value $\tilde{\varphi}_{0} \sim z^{4}$. According to the equation of motion (7) this is achieved if $m_{\varphi}^{2} R^{2}=0$ and for the canonical dimension of $\tilde{\varphi}$ we obtain from (3) the value $\Delta=4$. One can construct only one operator with $\Delta=4$ in the chiral limit: $G_{\mu \nu}^{a} G^{\mu \nu, a}$. In QCD, it acquires a vacuum expectation value - the gluon condensate $\left\langle G^{2}\right\rangle$ - that causes the scale anomaly in the trace of energy-momentum tensor. Since the fifth coordinate $z$ has the physical meaning of inverse energy scale, the condensation of the scalar field $\tilde{\varphi}$ is dual to the emergence of non-trivial gluon condensate in QCD - both effects make different energy scales non-equivalent. The fact that the anomaly is a quantum effect while in the holographic model the breaking of the scale invariance looks spontaneous should not be surprising as long as the dual theory is supposed to describe semiclassically the quantum effects in the strong coupling regime. Note also that the field $\tilde{\varphi}$ is in one-to-one correspondence with the dilaton field considered in the original paper [1] for demonstration of the idea of AdS/CFT correspondence.

According to an AdS/CFT prescription [25], the solution of classical equation of motion for a scalar field $\Phi$ corresponding to an operator $O$ has the following form near the $4 \mathrm{D}$ boundary $z \rightarrow 0$,

$$
\Phi(x, z) \rightarrow z^{4-\Delta}\left[\Phi_{0}(x)+\mathcal{O}\left(z^{2}\right)\right]+z^{\Delta}\left[\frac{\langle O(x)\rangle}{2 \Delta-4}+\mathcal{O}\left(z^{2}\right)\right],
$$

where $\Phi_{0}(x)$ acts as a source for $O(x)$ and $\langle O(x)\rangle$ denotes the corresponding condensate. Thus one has $\Lambda^{4} \sim\left\langle G^{2}\right\rangle$ in the case of the model (8). The exact solution for $\tilde{\varphi}_{0}$,

$$
\tilde{\varphi}_{0}(z)=C_{1}+C_{2} z^{4}
$$

has the form (9). The constant $C_{2}$ dictates the slope of the scalar trajectory, $C_{2} \lambda R^{2}=\Lambda^{4}$, while the constant term $\lambda C_{1}$ renormalizes the $5 \mathrm{D}$ mass $m_{s}$ in 
the action (8). It is curious to note that the $\operatorname{dim} 2$ condensate is not a well defined object in the relation (9).

\section{Mass spectrum and matching to OPE}

The mass spectrum of vector particles can be calculated following the standard procedure [3] by solving the equation of motion for the transverse components of the field $V_{\mu}$ in the action (6) or (5) (the gauge $V_{z}=0$ is implied),

$$
\left[-\frac{q^{2} V_{\mu}(q, z)}{z}-\partial_{z}\left(\frac{\partial_{z} V_{\mu}(q, z)}{z}\right)+\Lambda^{4} z V_{\mu}(q, z)\right]_{\perp}=0
$$

where $V_{\mu}(q, z)$ is the $4 \mathrm{D}$ Fourier transform of $V_{\mu}(x, z)$. By assumption, the physical particles correspond to the normalizable solutions of Eq. (11). According to the Sturm-Liouville theorem, such solutions $v_{n}(z)$ exist only for discrete values of $4 \mathrm{D}$ momentum $q^{2}$ which are identified with the on-shell masses $m_{n}^{2}$,

$$
-\partial_{z}\left(z^{-1} \partial_{z} v_{n}\right)+\Lambda^{4} z v_{n}=z^{-1} m_{n}^{2} v_{n} .
$$

The corresponding solutions are

$$
\begin{gathered}
m_{n}^{2}=4 \Lambda^{2}(n+1), \\
v_{n}(z)=\sqrt{\frac{2 n !}{(n+1) !}} e^{-z^{2} \Lambda^{2} / 2} z^{2} \Lambda^{2} L_{n}^{1}\left(z^{2} \Lambda^{2}\right) .
\end{gathered}
$$

Here $L_{n}^{1}$ denotes an associated Laguerre polynomial. The spectrum (13) coincides with the spectrum of the $\mathrm{SW}^{ \pm}$models but the exponential factor in the eigen-function (14) differs.

The alternative way for finding the spectrum is to calculate the vector two-point correlator and find its poles. We will show this calculation in detail in order to demonstrate the arising difference between the no-wall model and the SW one.

The two-point correlation function of vector currents $J_{\mu}$ is defined as

$$
\int d^{4} x e^{i q x}\left\langle J_{\mu}(x) J_{\nu}(0)\right\rangle=\left(q_{\mu} q_{\nu}-q^{2} g_{\mu \nu}\right) \Pi_{V}\left(Q^{2}\right), \quad Q^{2}=-q^{2} .
$$

According to the AdS/CFT prescription [1,2], the vector two-point correlator is given by the following relation [3],

$$
\Pi_{V}\left(Q^{2}\right)=-\frac{R}{g_{5}^{2} Q^{2}}\left[V(Q, z) \frac{\partial_{z} V(Q, z)}{z}\right]_{z \rightarrow 0},
$$


where $V(Q, z)$ stems from the $4 \mathrm{D}$ Fourier transform $V^{M}(Q, z)=V(Q, z) V_{0}^{M}(Q)$ for the $4 \mathrm{D}$ transverse part of $V^{M}$ and can be found from the equation of motion,

$$
-\partial_{z}\left(\frac{\partial_{z} V}{z}\right)+\Lambda^{4} z V=-\frac{Q^{2}}{z} V
$$

with the boundary conditions,

$$
V(Q, 0)=1, \quad V(Q, \infty)=0 .
$$

The solution is

$$
V(Q, z)=\Gamma\left(1+\frac{Q^{2}}{4 \Lambda^{2}}\right) e^{-z^{2} \Lambda^{2} / 2} U\left(\frac{Q^{2}}{4 \Lambda^{2}}, 0 ; z^{2} \Lambda^{2}\right),
$$

where $U$ is the Tricomi confluent hypergeometric function. This solution differs from the corresponding expression in the SW model by the exponential factor: In the $\mathrm{SW}^{-}$model, this factor is absent while in the $\mathrm{SW}^{+}$one the factor is $e^{-z^{2} \Lambda^{2}}$. Thus, the vector "bulk-to-boundary propagator" $V(Q, z)$ of the no-wall holographic model represents the logarithmic mean of the corresponding propagators of $\mathrm{SW}^{-}$and $\mathrm{SW}^{+}$models. The same property takes place for the wave functions of $4 \mathrm{D}$ discrete modes (14). Near the boundary, the solution (19) has the following expansion,

$$
\begin{gathered}
V(Q, z)_{z \rightarrow 0}=\left(1-\frac{z^{2} \Lambda^{2}}{2}+\mathcal{O}\left(z^{4} \Lambda^{4}\right)\right) \times \\
\left\{1+\frac{Q^{2}}{4 \Lambda^{2}}\left[\log \left(z^{2} \Lambda^{2}\right)+\psi\left(1+\frac{Q^{2}}{4 \Lambda^{2}}\right)+2 \gamma-1\right] z^{2} \Lambda^{2}+\mathcal{O}\left(z^{4} \Lambda^{4}\right)\right\}= \\
1+\left\{\frac{Q^{2}}{4 \Lambda^{2}}\left[\log \left(z^{2} \Lambda^{2}\right)+\psi\left(1+\frac{Q^{2}}{4 \Lambda^{2}}\right)+2 \gamma-1\right]-\frac{1}{2}\right\} z^{2} \Lambda^{2}+\mathcal{O}\left(z^{4} \Lambda^{4}\right),
\end{gathered}
$$

where the expression in the first line emerges from the exponential factor in (19). The Digamma function can be represented as

$$
\psi\left(1+\frac{Q^{2}}{4 \Lambda^{2}}\right)=\sum_{k=1}^{\infty} \frac{1}{k}-\sum_{k=1}^{\infty} \frac{1}{\frac{Q^{2}}{4 \Lambda^{2}}+k}-\gamma .
$$

Substituting now the expansion (20) into the relation (16) and exploiting the representation (21), after subtracting the contact terms and redefining $k=n+1$ we arrive at our final answer for the vector correlator,

$$
\Pi_{V}\left(Q^{2}\right)=\frac{R}{2 g_{5}^{2}}\left[\sum_{n=0}^{\infty} \frac{4 \Lambda^{2}}{Q^{2}+4 \Lambda^{2}(n+1)}+\frac{2 \Lambda^{2}}{Q^{2}}\right] .
$$


The poles $Q_{n}^{2}=-m_{n}^{2}$ in the sum of expression (22) yield the mass spectrum (13). As was said above, this spectrum coincides with the spectrum of $\mathrm{SW}^{ \pm}$model for the vector mesons.

The massless pole that is present in the correlator turns out to be indispensable for providing the correct high energy asymptotic behavior. Making use of the expansion

$$
\psi\left(1+\frac{Q^{2}}{4 \Lambda^{2}}\right)_{Q^{2} \rightarrow \infty}=\log \left(\frac{Q^{2}}{4 \Lambda^{2}}\right)+\frac{2 \Lambda^{2}}{Q^{2}}-\frac{4 \Lambda^{4}}{3 Q^{4}}+\mathcal{O}\left(\frac{\Lambda^{8}}{Q^{8}}\right)
$$

we obtain the following asymptotic expansion of the correlator under consideration,

$$
\Pi_{V}\left(Q^{2}\right)_{Q^{2} \rightarrow \infty}=\frac{R}{2 g_{5}^{2}}\left[\log \left(\frac{4 \Lambda^{2}}{Q^{2}}\right)+\frac{4 \Lambda^{4}}{3 Q^{4}}+\mathcal{O}\left(\frac{\Lambda^{8}}{Q^{8}}\right)\right] .
$$

The expansion (24) can be matched to the Operator Product Expansion (OPE) for the vector two-point correlator [26],

$$
\Pi_{V}\left(Q^{2}\right)_{Q^{2} \rightarrow \infty}=\frac{N_{c}}{24 \pi^{2}} \log \left(\frac{\mu_{\mathrm{ren}}^{2}}{Q^{2}}\right)+\frac{\alpha_{s}}{24 \pi} \frac{\left\langle G^{2}\right\rangle}{Q^{4}}+\mathcal{O}\left(\frac{\mu_{\mathrm{ren}}^{6}}{Q^{6}}\right) .
$$

The matching of coefficients in front of the logarithmic terms yields the standard normalization factor for the 5D vector field,

$$
\frac{R}{g_{5}^{2}}=\frac{N_{c}}{12 \pi^{2}}
$$

An important feature of the no-wall model is the absence of $\mathcal{O}\left(Q^{-2}\right)$ contribution in the expansion (24) as required by the OPE (25) - in QCD in the chiral limit, there is no local gauge invariant condensate of dimension two. The cancelation of $\mathcal{O}\left(Q^{-2}\right)$ contribution occurs due to the massless pole in the correlator (22). Looking at the expansion (20) it is easy to notice that the pole term stems from the exponential factor. As follows from the remark after the relation (19) the pole term is absent in the $\mathrm{SW}^{-}$model and is twice the pole term of (22) in the $\mathrm{SW}^{+}$model. Thus, both the $\mathrm{SW}^{-}$and the $\mathrm{SW}^{+}$ models possess a feature that we do not expect in QCD - they predict a non-zero dimension-two condensate in the OPE,

$$
\Pi_{V}\left(Q^{2}\right)_{Q^{2} \rightarrow \infty}^{\mathrm{SW}^{ \pm}}=\frac{R}{2 g_{5}^{2}}\left[\log \left(\frac{4 \Lambda^{2}}{Q^{2}}\right) \pm \frac{2 \Lambda^{2}}{Q^{2}}+\mathcal{O}\left(Q^{-4}\right)\right] .
$$

The dimension-two condensate of the no-wall model represents the arithmetic mean of the dimension-two condensates of the $\mathrm{SW}^{+}$and the $\mathrm{SW}^{-}$models and as seen from (27) this very property cancels the $\mathcal{O}\left(Q^{-2}\right)$ contribution. 


\section{Conclusions}

We have proposed a new type of bottom-up holographic model describing the Regge-like spectrum of the radially excited states. In contrast to the soft wall models, the considered setup does not contain the dilaton background. The introduced dynamical mechanism for mass generation is not novel - it is widely exploited for description of the chiral symmetry breaking in the holographic approach. The spontaneous chiral symmetry breaking contributes to the masses of low-lying states but it is definitely not dominant in the generation of masses of highly excited resonances. Within the presented scheme, a similar mechanism provides the main contribution to the masses of all light mesons from a dual description of gluodynamics. The distinguishing features of the no-wall model with respect to the soft wall one are: The physical interpretation is more transparent (e.g., the appearance of discrete meson spectrum and its Regge form are directly related to the formation of gluon condensates in QCD), the absence of dimension-two condensate in the power expansion of the two-point correlators, the model is closer to the original AdS/CFT theoretical setup (the pure AdS space in the bulk), and the exploited Higgs-like mechanism for mass generation looks more natural for the field-theoretical description.

The most important lesson of the present work is that the effective presence of $\operatorname{dim} 2$ operator cannot be removed from the holographic models describing the Regge like spectrum: If we replace the soft wall model by the no-wall one and remove thereby the $\operatorname{dim} 2$ condensate from the power expansion of the vector correlators then this contribution shows up directly in the action in the form of a scalar field corresponding to the dim2 operator.

The scalar fields which generates the mass spectrum in the no-wall model correspond to the gluon operators. Since the gluon sector is involved one should analyze the back reaction of the metric in response to the condensation of these fields. The given work is left for future.

The properties of the no-wall holographic model suggest that this model represents an alternative possibility for constructing five-dimensional dual descriptions for QCD.

\section{Acknowledgments}

I am grateful to my colleagues from the Department of Theoretical Physics of Saint-Petersburg State University for fruitful discussions. The work is supported by RFBR, grant 09-02-00073-a, by SPbSU grant 11.0.64.2010, by the Alexander von Humboldt Foundation (Return Fellowship) and by the 
Dynasty Foundation.

\section{References}

[1] E. Witten, Adv. Theor. Math. Phys. 2, 253 (1998).

[2] S. S. Gubser, I. R. Klebanov and A. M. Polyakov, Phys. Lett. B 428, 105 (1998).

[3] J. Erlich, E. Katz, D. T. Son and M. A. Stephanov, Phys. Rev. Lett. 95, 261602 (2005).

[4] L. Da Rold and A. Pomarol, Nucl. Phys. B 721, 79 (2005).

[5] A. Karch, E. Katz, D. T. Son and M. A. Stephanov, Phys. Rev. D 74, 015005 (2006).

[6] O. Andreev, Phys. Rev. D 73, 107901 (2006).

[7] H. Boschi-Filho, N. R. F. Braga and H. L. Carrion, Phys. Rev. D 73, 047901 (2006).

[8] J. Hirn, N. Rius and V. Sanz, Phys. Rev. D 73, 085005 (2006).

[9] K. Ghoroku, N. Maru, M. Tachibana and M. Yahiro, Phys. Lett. B 633, $602(2006)$.

[10] C. Csáki and M. Reece, JHEP 0705, 062 (2007).

[11] J. P. Shock, F. Wu, Y.-L. Wu and Z.-F. Xie, JHEP 0703, 064 (2007).

[12] B. Batell and T. Gherghetta, Phys. Rev. D 78, 026002 (2008).

[13] P. Colangelo, F. De Fazio, F. Giannuzzi, F. Jugeau and S. Nicotri, Phys. Rev. D 78, 055009 (2008).

[14] W. de Paula, T. Frederico, H. Forkel and M. Beyer, Phys. Rev. D 79, 075019 (2009).

[15] A. Vega and I. Schmidt, Phys. Rev. D 79, 055003 (2009).

[16] T. Gherghetta, J. I. Kapusta and T. M. Kelley, Phys. Rev. D 79, 076003 (2009).

[17] S. S. Afonin, Phys. Lett. B 675, 54 (2009). 
[18] S. S. Afonin, Phys. Lett. B 678, 477 (2009).

[19] G. F. de Teramond and S. J. Brodsky, Phys. Rev. Lett. 102, 081601 (2009).

[20] A. Cherman, T. D. Cohen and E. S. Werbos, Phys. Rev. C 79, 045203 (2009).

[21] F. Zuo, Phys. Rev. D 82, 086011 (2010).

[22] D. Becciolini, M. Redi and A. Wulzer, JHEP 1001 (2010) 074.

[23] S. S. Afonin, Int. J. Mod. Phys. A 25, 5683 (2010).

[24] F. V. Gubarev and V. I. Zakharov, Phys. Lett. B 501, 28 (2001); K. G. Chetirkin, S. Narison, and V. I. Zakharov, Nucl. Phys. B 550, 353 (1999); K.-I. Kondo, Phys. Lett. B 514, 335 (2001); H. Verschelde, K. Knecht, K. Van Acoleyen, and M. Vanderkelen, Phys. Lett. B 516, 307 (2001); P. Boucaud et al., Phys. Rev. D 63, 114003 (2001); E. Ruiz Arriola, P. O. Bowman, and W. Broniowski, Phys. Rev. D 70, 097505 (2004); E. Megias, E. Ruiz Arriola, and L. L. Salcedo, JHEP 01, 073 (2006); V. I. Zakharov, hep-ph/0509114; S. Narison, hep-ph/0508259; E. Ruiz Arriola and W. Broniowski, Phys. Rev. D 73, 097502 (2006); 81, 054009 (2010).

[25] I. R. Klebanov and E. Witten, Nucl. Phys. B 556, 89 (1999).

[26] M. A. Shifman, A. I. Vainstein and V. I. Zakharov, Nucl. Phys. B 147, 385,448 (1979). 